02

\title{
Определение коэффициента экстинкции моноксида углерода, адсорбированного на диоксиде титана
}

\author{
(C) К.М. Буланин ${ }^{1,2}$, А.Ю. Михелева ${ }^{2}$, Д.Н. Щепкин ${ }^{3}$, А.В. Рудакова ${ }^{2}$ \\ ${ }^{1}$ Санкт-Петербургский государственный университет, кафедра фотоники, \\ 199034 Санкт-Петербург, Россия \\ ${ }^{2}$ Санкт-Петербургский государственный университет, лаборатория „Фотоактивные нанокомпозитные материалы“, \\ 199034 Санкт-Петербург, Россия \\ ${ }^{3}$ Санкт-Петербургский государственный университет, кафедра молекулярной спектроскопии, \\ 199034 Санкт-Петербург, Россия \\ e-mail: k.bulanin@spbu.ru
}

Поступила в редакцию 02.07.2021 г.

В окончательной редакции 02.07.2021 г.

Принята к публикации 26.07.2021 г.

\begin{abstract}
Изучена адсорбция моноксида углерода на поверхности $\mathrm{TiO}_{2}$ (анатаз) при комнатной температуре методами ИК спектроскопии и волюметрии. Полученные экспериментальные данные указывают на слабую адсорбцию молекул СО на экспоненциально-неоднородной поверхности. Установлено, что теплота адсорбции уменьшается по логарифмическому закону с покрытием в диапазоне $0.002-0.03$. Рассчитан коэффициент экстинкции адсорбированного $\mathrm{CO}$ для гетерогенной системы $\mathrm{CO} / \mathrm{TiO}_{2}$ с использованием закона Бугера-Ламберта-Бера и с учетом поправки на поле Лоренца. Описаны условия правомерности применимости закона Бугера-Ламберта-Бера для изучения гетерогенных систем.
\end{abstract}

Ключевые слова: оксид углерода (II), диоксид титана, коэффициент экстинкции, адсорбция, ИК спектроскопия, волюметрия.

DOI: 10.21883/OS.2021.11.51639.2511-21

\section{Введение}

Статья посвящается 90-летию со дня рождения профессора Михаила Олеговича Буланина.

Определение силы, типа и количества адсорбционных центров на поверхности порошкообразных катализаторов с помощью ИК спектроскопии адсорбированных тест-молекул является эффективным методом характеризации их поверхностных свойств $[1,2]$.

Одной из наиболее широко применяемых тест-молекул является моноксид углерода. Являясь слабым основанием Льюиса, молекула СО обладает слабовыраженным электронодонорным свойством. Так, было установлено, что при адсорбции на поверхности оксидов металлов данная молекула взаимодействует с поверхностными катионными центрами, как правило, через атом углерода. Однако в некоторых случаях взаимодействие с катионом переходных металлов также возможно через ее атом кислорода [3]. При адсорбции через атом углерода наблюдается увеличение частоты валентного колебания $\mathrm{C}=\mathrm{O}$ по сравнению с таковой для молекулы в газовой фазе. При этом было экспериментально установлено, что величина частотного сдвига адсорбата по сравнению с невозмущенной взаимодействием молекулой может быть использована для оценки силы адсорбционных центров [4].

Для сравнительной количественной характеризации адсорбционной и каталитической эффективности различных материалов обычно используют концентрацию их активных центров, которую можно экспериментально определить, зная интегральный коэффициент экстинкции адсорбированной тест-молекулы.

Известно, что коэффициент экстинкции свободной молекулы определяется вероятностью ее перехода из основного в возбужденное состояние, которая должна изменяться в результате ее взаимодействия с поверхностью. Вместе с тем исследованию влияния адсорбции СО на поверхности твердых тел (в том числе на поверхности такого широко применяемого в катализе и фотокатализе материала как диоксид титана) на величину его коэффициента экстинкции посвящено лишь незначительное количество работ $[5,6]$.

Так, в работе [5] были измерены коэффициенты экстинкции моноксида углерода, адсорбированного при $77 \mathrm{~K}$ на различных мелкодисперсных адсорбентах, включая $\mathrm{TiO}_{2}$. Полученная экспериментальная зависимость интегрального коэффициента поглощения адсорбированного $\mathrm{CO}$ от частоты ее валентного колебания для ряда исследуемых адсорбентов рассмотрена в рамках электростатической модели, основанной на эффекте Штарка.

В работе [6] ИК спектроскопическое исследование адсорбции СО на различных кристаллографических модификациях диоксида титана было проведено при комнатной температуре при равновесных давлениях адсорбата вплоть до 150 Torr. Величина покрытия поверхности адсорбатом достигала величин до $100 \mu \mathrm{mol} / \mathrm{g}$. Значения коэффициентов экстинкции $\mathrm{CO}$, адсорбированного на различных образцах $\mathrm{TiO}_{2}$, определялись с использовани- 
ем закона Бугера-Ламберта-Бера. Были представлены корреляционные зависимости коэффициентов экстинкции молекул $\mathrm{CO}$, адсорбированных на различных типах центров, и соответствующим им положения максимумов ИК полос. Наряду с этим было отмечено влияние рассеивающих характеристик адсорбента на определяемую величину коэффициента экстинкции адсорбированных молекул, что ограничивает применимость закона Бугера-Ламберта-Бера к гетерогенным и сильно рассеивающим системам.

В настоящей работе была исследована адсорбция $\mathrm{CO}$ на $\mathrm{TiO}_{2}$ модификации анатаз при комнатной температуре методом ИК спектроскопии. Целью работы являлось определение коэффициента экстинкции СО в адсорбированном состоянии при малых покрытиях. Проведено сравнение полученного коэффициента экстинкции с известными из литературы данными. Обнаружена зависимость исследуемого параметра от величины покрытия поверхности адсорбатом, что связано с энергетической неоднородностью поверхности. Сформулированы факторы, влияющие на значение коэффициента экстинкции молекул в адсорбированном состоянии, в том числе при применении закона Бугера-Ламберта-Бера для рассеивающих систем.

\section{Экспериментальная часть}

Исследуемый в работе образец представляет собой порошок диоксида титана (анатаз) марки Hombikat UV100 (Sachtleben Chemie GmbH, Германия). Удельная поверхность порошка составляла $300 \mathrm{~m}^{2} / \mathrm{g}$, характерный размер кристаллитов $20-40 \mathrm{~nm}$.

Для ИК спектроскопических исследований в режиме пропускания образец был спрессован в виде таблетки с размерами $11.5 \times 11.0 \mathrm{~mm}$ и толщиной $0.135 \mathrm{~mm}$, при этом его масса составляла 0.025 g. На рис. 1 представлена микрофотография спрессованного образца.

Полученную таблетку закрепляли в специальном держателе для образца, который помещали в кювету для спектроскопических исследований адсорбированных молекул, подробно описанную в работе [7]. Кратко отметим, что данное устройство позволяет регистрировать спектры порошкообразных образцов при комнатной температуре в вакууме и в присутствии различных газов. Давление в системе напуска и в рабочем объеме контролировалось с помощью емкостных манометров CTR100 (Oerlikon Leybold Vacuum, Германия) с диапазонами измеряемых давлений до 10 и 1000 Torr. Вакуум в системе контролировался с помощью вакуумметра с холодным катодом AIM-X-MSEAL (Edwards, Великобритания), позволяющего измерять вакуум до $\sim 10^{-9}$ Torr.

Для очистки от органических загрязнителей исходный образец подвергался прогреву при $500^{\circ} \mathrm{C}$ в вакууме ( $\sim 10^{-6}$ Torr $)$, затем в кислороде. В ходе проведения экспериментов по исследованию адсорбции моноксида

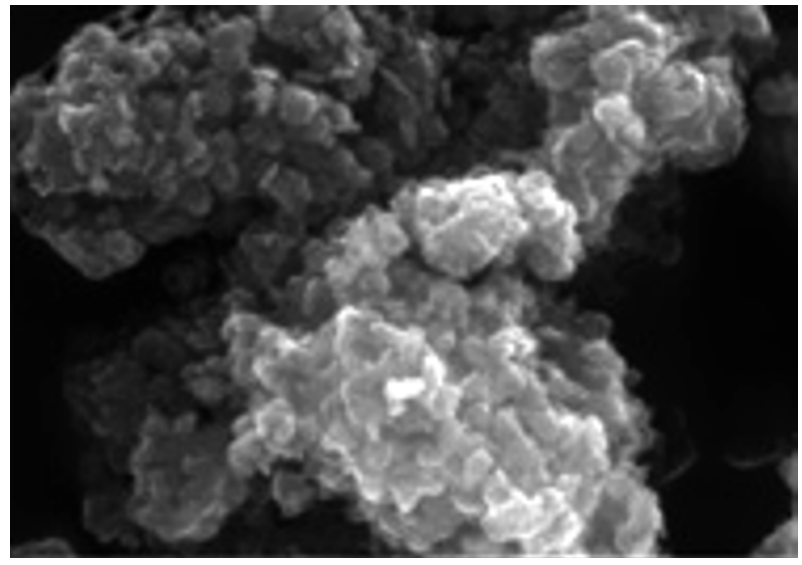

$100 \mathrm{~nm}$

Рис. 1. Изображение частиц спрессованного образца диоксида титана, полученное с помощью сканирующего электронного микроскопа.

углерода перед каждым напуском адсорбата образец подвергался термовакуумной обработке при температуре $450^{\circ} \mathrm{C}$ в вакууме, далее для получения одного и того же окисленного состояния охлаждался до комнатной температуры в присутствии газообразного кислорода.

В работе применялись газы высокой чистоты кислород (Linde, 99.999\%) и оксид углерода (II) (Linde, 99.99\%). Диапазон используемых равновесных давлений адсорбата находился в пределах от 0.04 до 6.00 Torr.

ИК спектры поглощения были зарегистрированы с помощью ИК спектрометра Thermo Nicolet iS50 (Thermo Scientific, США) в спектральном диапазоне $1000-8000 \mathrm{~cm}^{-1}$ со спектральным разрешением $4 \mathrm{~cm}^{-1}$. Для уменьшения влияния на образец термического воздействия ИК излучения от источника прибора перед кюветой с образцом устанавливалась ослабляющая сетка. Обработка спектральных данных осуществлялась в программных пакетах Omnic 7.3, Origin 9.0 и Fityk 1.3.1.

Количество молей молекул $\mathrm{CO}$, адсорбированных на всем образце диоксида титана, обозначаемое в дальнейшем $n$, определяли волюметрическим методом. Используя известные объемы измерительной и рабочей частей установки, с помощью уравнения Менделеева-Клапейрона рассчитывали количество молей адсорбата как в отсутствие, так и в присутствии адсорбента при установлении адсорбционного равновесия (60 min после напуска газа) при комнатной температуре $\left(23^{\circ} \mathrm{C}\right)$. Разность числа молей газа, отнесенная к единице массы адсорбента, соответствовала величине адсорбции моноксида углерода на исследуемом образце при соответствующем равновесном давлении адсорбата.

Для оценки интегрального коэффициента экстинкции количество адсорбированного газа, соответствующее спектроскопически анализируемому количеству адсорбента, обозначаемое далее как $n_{\text {ads }}$, рассчитывалось по 
формуле

$$
n_{\mathrm{ads}}=\frac{k \pi d^{2} l}{4 a b l} n=\frac{k \pi d^{2}}{4 a b} n=0.0373 n,
$$

где $k$ - коэффициент ослабляющей сетки, равный 0.06 , $d$ - диаметр анализирующего пучка прибора на образце, равный $10 \mathrm{~mm}$, величины $a, b$ и $l-$ соответственно ширина, высота и толщина исследуемого образца.

\section{Результаты и обсуждение}

\section{Исследование адсорбции СО на поверхности диоксида} титана

На рис. 2, $a$ представлены спектры моноксида углерода, адсорбированного при комнатной температуре на поверхности диоксида титана, предварительно дегидратированного при $450^{\circ} \mathrm{C}$, при различных равновесных давлениях адсорбата. Можно видеть, что при малых давлениях в спектре адсорбированного СО наблюдается преимущественно одна полоса поглощения с максимумом около $2210 \mathrm{~cm}^{-1}$, в то время как с увеличением давления появляется вторая полоса при $2193 \mathrm{~cm}^{-1}$. При этом вместе с увеличением покрытия поверхности увеличиваются интенсивности обеих полос, а их максимумы сдвигаются в сторону низких частот: с 2210 к $2208 \mathrm{~cm}^{-1}$ и с 2193 к $2191 \mathrm{~cm}^{-1}$ соответственно. Полученные ИК спектры адсорбированных на поверхности $\mathrm{TiO}_{2}$ молекул СО были использованы для нахождения интегральных интенсивностей $(A)$ : как суммарной для обеих полос $\left(A_{\text {sum }}\right)$ в спектральном интервале $2250-2130 \mathrm{~cm}^{-1}$, так и для каждой из наблюдаемых ИК полос поглощения $\left(A_{2210}\right.$ и $\left.A_{2190}\right)$, полученных разделением на компоненты с использованием функции Фойгта. На рис. $2, b$ представлены зависимости этих параметров от равновесного давления СО. Можно отметить тенденцию к насыщению интенсивности высокочастотной полосы с ростом покрытия.

Известно, что при комнатной температуре моноксид углерода адсорбируется на поверхности диоксида титана достаточно слабо $[5,8,9]$. Ранее сообщалось $[5,9]$, что присутствие в ИК спектре двух полос поглощения (рис. 2, $a$ ) указывает на то, что адсорбция происходит на двух типах электроноакцепторных центров (кислотных центров Льюиса) на поверхности исследуемого образца. Такие поверхностные центры представляют собой катионы титана $\mathrm{Ti}^{4+}$ с низкой степенью координации (преимущественно катионы на ребрах кристаллитов с координационными числом, равным 5) [10]. В этом случае молекулы СО образуют координационные связи с катионами через атом углерода посредством $\sigma$-донирования [9,11-13].

Экспериментальные данные волюметрического определения количества адсорбированного газа (см. раздел Экспериментальная часть), а также найденные интегральные интенсивности полос поглощения представлены в табл. 1.
Таблица 1. Экспериментальные данные по адсорбции СО на $\mathrm{TiO}_{2}$ при $23^{\circ} \mathrm{C}$ : количество молей адсорбата на грамм адсорбента $\left(n_{\text {ads }}\right)$ при соответствующем равновесном давлении адсорбата $(p)$, степень заполнения $(\theta)$, интегральные интенсивности (суммарная $\left(A_{\text {sum }}\right)$ для обеих полос и для отдельных полос $\left(A_{2210}\right.$ и $\left.\left.A_{2190}\right)\right)$

\begin{tabular}{r|c|c|c|c|c|c}
\hline № & $\begin{array}{c}p, \\
\text { Torr }\end{array}$ & $\begin{array}{c}n_{\text {ads, }}, \\
\mu \mathrm{mol} / \mathrm{g}\end{array}$ & $\theta^{*}$ & $\begin{array}{c}A_{\text {sum, }}, \\
\mathrm{cm}^{-1}\end{array}$ & $\begin{array}{c}A_{2210}, \\
\mathrm{~cm}^{-1}\end{array}$ & $\begin{array}{c}A_{2193}, \\
\mathrm{~cm}^{-1}\end{array}$ \\
\hline 1 & 0.0405 & 4.830 & 0.002 & 0.528 & 0.440 & 0.064 \\
2 & 0.0865 & 8.122 & 0.003 & 0.852 & 0.793 & 0.150 \\
3 & 0.1900 & 11.384 & 0.005 & 1.201 & 0.949 & 0.240 \\
4 & 0.3878 & 15.794 & 0.006 & 1.577 & 1.116 & 0.408 \\
5 & 0.7128 & 21.942 & 0.009 & 2.156 & 1.297 & 0.712 \\
6 & 1.4297 & 31.533 & 0.013 & 2.820 & 1.462 & 1.200 \\
7 & 1.9250 & 35.422 & 0.014 & 2.973 & 1.500 & 1.480 \\
8 & 2.4159 & 39.512 & 0.016 & 3.280 & 1.510 & 1.690 \\
9 & 2.9347 & 45.404 & 0.018 & 3.507 & 1.594 & 1.879 \\
10 & 3.6386 & 56.178 & 0.022 & 4.200 & 1.700 & 2.300 \\
11 & 4.1320 & 62.056 & 0.025 & 4.510 & 1.730 & 2.450 \\
12 & 4.9320 & 64.793 & 0.026 & 4.660 & 1.951 & 2.557 \\
13 & 5.9607 & 68.582 & 0.028 & 5.086 & 1.951 & 2.928
\end{tabular}

Примечание. * Степень заполнения поверхности $(\theta)$ адсорбированными молекулами была рассчитана в предположении, что максимальное количество доступных центров на $1 \mathrm{~cm}^{2}$ поверхности составляет $5 \cdot 10^{14}[14,15]$.

На рис. 3, $a$ представлена изотерма адсорбции молекул моноксида углерода на поверхности $\mathrm{TiO}_{2}$ при комнатной температуре, построенная по полученным экспериментальным данным. При давлениях адсорбата до 6 Torr количество адсорбированного газа постепенно увеличивается, однако не достигает максимального покрытия, что не позволяет отнести данную зависимость к изотерме Ленгмюра, хорошо описывающей адсорбцию на энергетически однородных поверхностях [16].

Анализ зависимостей интегральных коэффициентов поглощения отдельных полос адсорбированного $\mathrm{CO}$ от равновесного давления адсорбата (рис. $2, b$ ) позволяет сделать вывод, что заполнение доступных адсорбционных центров первого типа (им соответствует высокочастотная полоса при $2210 \mathrm{~cm}^{-1}$ ) приходит к насыщению при давлениях адсорбата, близких к 2 Torr, в то время как адсорбция $\mathrm{CO}$ на более слабых центрах второго типа (полоса при $2193 \mathrm{~cm}^{-1}$ ) продолжается вплоть до давления, равного 6 Torr. Аналогичные зависимости от покрытия интенсивностей высокочастотной и низкочастотной полос $\mathrm{CO}$, адсорбированного на $\mathrm{TiO}_{2}$, описаны в работе [9]. Таким образом, это указывает на то, что адсорбция СО на исследуемом образце анатаза происходит на энергетически неоднородной поверхности.

Для определения типа энергетической неоднородности поверхности полученные экспериментальные данные по адсорбции были представлены в виде двух зависимостей: $\theta=f(\ln p), \quad$ описывающей изотерму Темкина-Фрумкина для адсорбции на равномернонеоднородных поверхностях, и $\ln \theta=f(\ln p)$, 

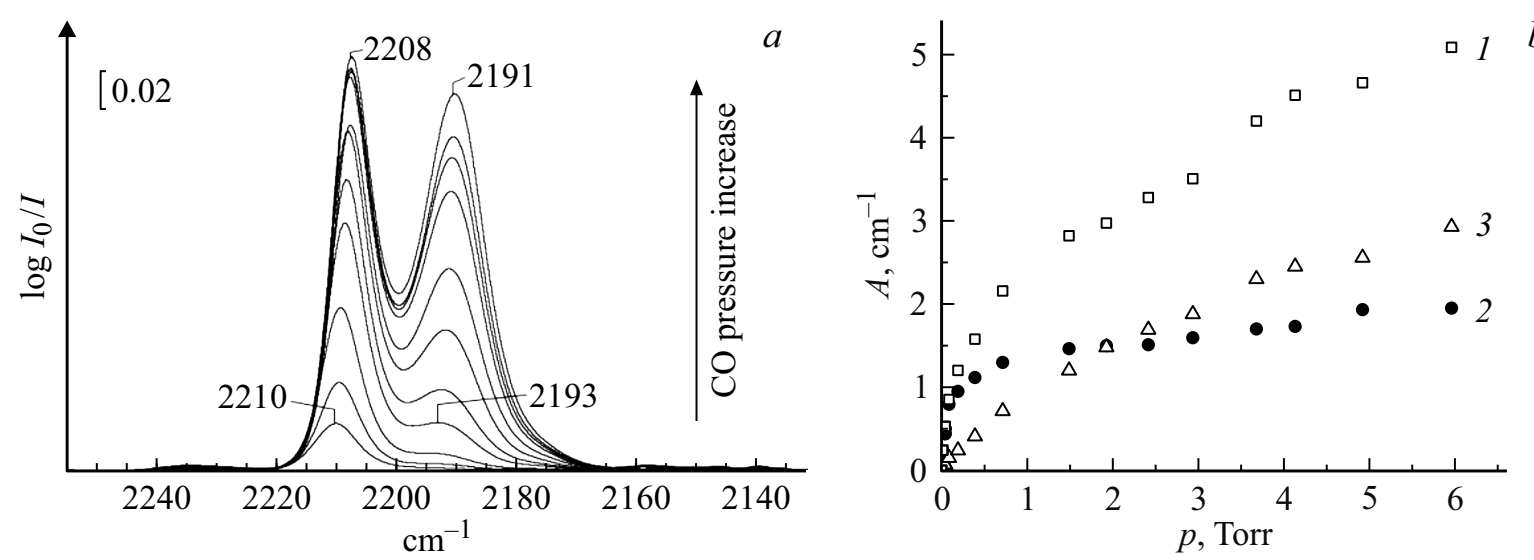

Рис. 2. (a) ИК спектры молекул $\mathrm{CO}$, адсорбированных на поверхности $\mathrm{TiO}_{2}$ при комнатной температуре в диапазоне давлений адсорбата от 0.04 Torr $(5.3 \mathrm{~Pa})$ до 6.00 Torr $(800 \mathrm{~Pa})$. Спектры регистрировались при комнатной температуре. (b) Зависимость интегральных интенсивностей полос поглощения адсорбированных молекул от равновесного давления: 1 - для обеих полос, $2-$ для полосы при $2210 \mathrm{~cm}^{-1}, 3$ - для полосы при $2193 \mathrm{~cm}^{-1}$.
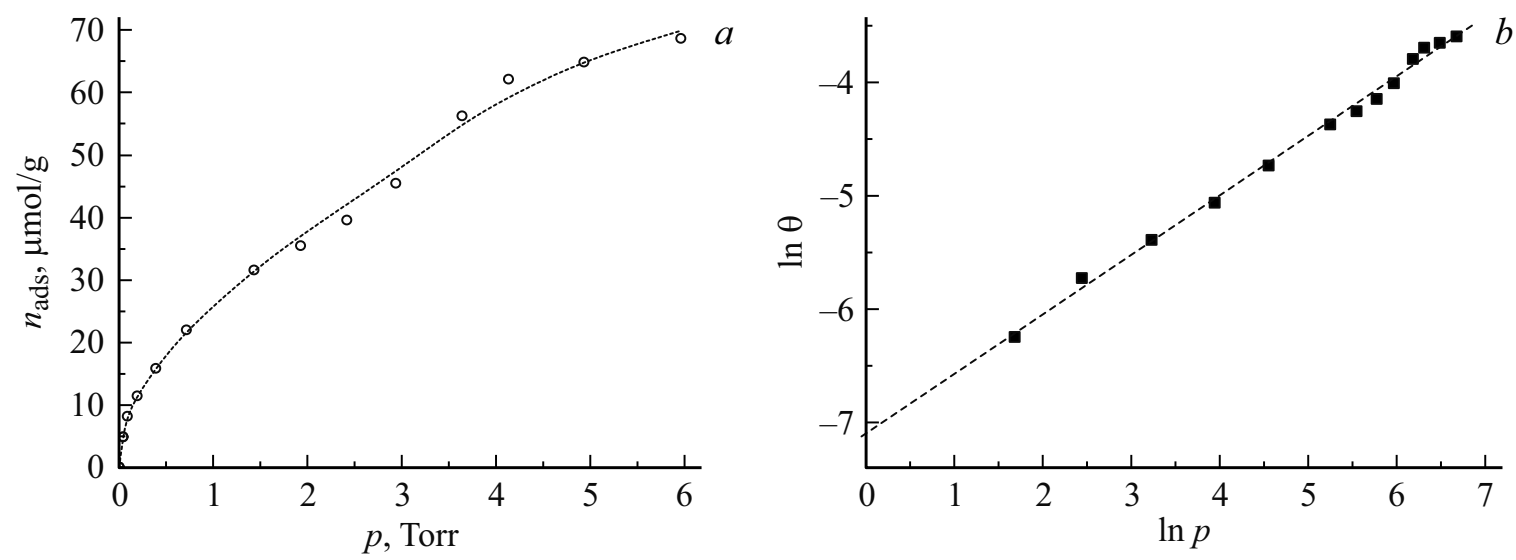

Рис. 3. Изотерма адсорбции моноксида углерода на поверхности диоксида титана, дегидратированного при $450^{\circ} \mathrm{C}$, в координатах: (a) $n_{\text {ads }}-p,(b) \ln \theta-\ln p$.

описывающей изотерму Фрейндлиха для адсорбции на экспоненциально-неоднородных поверхностях, где $\theta$ - степень заполнения поверхности адсорбатом (табл. 1) [16-19]. На рис. 3, $b$ представлены данные в координатах $\ln \theta-\ln p$, которые хорошо описываются прямолинейной функцией с высоким коэффициентом аппроксимации, равным 0.9945. Линейный вид именно этой зависимости позволяет сделать вывод, что экспериментальные данные по адсорбции хорошо описываются изотермой Фрейндлиха [16,17]:

$$
\theta=\beta p^{1 / \alpha}
$$

где $\beta$ - константа, представляющая собой по смыслу покрытие при давлении, равном $1 \mathrm{~Pa}, \alpha$ - параметр, показывающий отклонение от линейности зависимости покрытия от давления, $\alpha \geq 1$.

В этом случае распределение неоднородности поверхности по теплотам адсорбции для исследуемого образца диоксида титана после термообработки при $450^{\circ} \mathrm{C}$ носит экспоненциальный характер [19]. При этом зависимость теплоты адсорбции уменьшается с увеличением степени заполнения по логарифмическому закону:

$$
\Delta H=\Delta H_{0}-c \ln \theta,
$$

где $\Delta H-$ теплота адсорбции при степени заполнения $\theta$, $\Delta H_{0}$ - теплота адсорбции при $\theta=0, c-$ константа.

Из линейной аппроксимации зависимости $\ln \theta$ от $\ln p$, представленной на рис. $3, b$, отрезок, отсекаемый прямой на оси ординат, будет представлять собой величину $\ln \beta$, а тангенс угла наклона прямой к оси абсцисс величину $1 / \alpha$. Найденные значения $\beta$ и $\alpha$ оказались равными $8.25 \cdot 10^{-4}$ и 1.9 соответственно.

Теплота адсорбции может быть определена из эмпирической зависимости этой величины и частотного сдвига полосы поглощения $\mathrm{CO}$, адсорбированного на катионах элементов главной группы и некоторых переходных металлов, относительно полосы $\mathrm{CO}$ в газовой фазе $\left(\Delta v(\mathrm{CO})=v\left(\mathrm{CO}_{\mathrm{ads}}\right)-v\left(\mathrm{CO}_{\mathrm{g}}\right)\right.$, где $\left.v\left(\mathrm{CO}_{\mathrm{g}}\right)=2143 \mathrm{~cm}^{-1}\right)$, предложенной в работах [20-22]:

$$
\Delta H\left(\mathrm{~kJ} \cdot \mathrm{mol}^{-1}\right)=10.5+0.5 \Delta v(\mathrm{CO}) \text {. }
$$


C помощью выражения (4) была найдена теплота адсорбции при минимальном покрытии $\mathrm{CO}$, когда $\Delta v(\mathrm{CO})=67 \mathrm{~cm}^{-1}$. Полученное значение, равное $\Delta H_{0}^{2210}=44 \mathrm{~kJ} \cdot \mathrm{mol}^{-1}$, соответствует слабой адсорбции молекул $\mathrm{CO}$ на поверхности диоксида титана при комнатной температуре и хорошо согласуется с порядком этой величины на образцах диоксида титана, полученным различными экспериментальными и расчетными методами [5,9,14,22-25].

Дальнейшее увеличение покрытия (рис. 2,a) приводит к появлению второй полосы поглощения около $2190 \mathrm{~cm}^{-1}$, соответствующей молекулам СО, адсорбированным на более слабых поверхностных центрах с меньшей теплотой адсорбции, равной $\Delta H_{0}^{2190}=35.5 \mathrm{~kJ} \cdot \mathrm{mol}^{-1}$. При максимальном покрытии $(\theta=0.028)$, исследуемом в настоящей работе, наблюдался незначительный низкочастотный сдвиг обеих полос. Такой сдвиг можно было бы объяснить наличием резонансного диполь-дипольного взаимодействия между адсорбированными молекулами моноксида углерода [25]. Однако при очень низких степенях заполнения $\theta$ (от тысячных до сотых долей монослоя, табл. 1) обмен энергией между молекулами на поверхности практически отсутствует. Таким образом, низкочастотный сдвиг связан с наблюдаемым падением теплоты адсорбции при увеличении покрытия до 0.028 (для $\Delta H^{2210}$ - с 44 до $43 \mathrm{~kJ} \cdot \mathrm{mol}^{-1}$, для $\Delta H^{2190}$ - с 35.5 до $34.5 \mathrm{~kJ} \cdot \mathrm{mol}^{-1}$ ), что является ожидаемым фактом согласно выражению (3) и согласуется с литературными данными [9,10,23].

Коэфбициент экстинкиии СО, адсорбированного на поверхности диоксида титана

Для нахождения интегрального коэффициента экстинкции СО, адсорбированного на поверхности диоксида титана, использовался закон Бугера-Ламберта-Бера как в ранее опубликованных работах [5,21,26-32]:

$$
I_{v}=I_{0 v} e^{-C x \varepsilon}
$$

где $I_{0 v}$ и $I_{v}-$ интенсивности падающего и прошедшего света, $x$ - оптический путь, $\varepsilon-$ коэффициент экстинкции, $C=\frac{n_{\mathrm{ads}}}{V}-$ концентрация адсорбата в анализируемом пучком излучения прибора объеме образца $V=\frac{k \pi d^{2} l}{4}$ в пятне света диаметром $d(10 \mathrm{~mm})$.

Далее все численные значения известных величин подставляли в уравнение (5), учитывая, что измеряемая ИК спектрометром интенсивность полос адсорбированного СО равна

$$
A=\int_{0}^{\infty} \log \frac{I_{0 v}}{I_{v}} d v
$$

и, принимая оптический путь равным толщине исследуемого образца $l(0.0135 \mathrm{~cm})$, получали выражение для $\varepsilon$ адсорбированного СО в единицах измерения $\mathrm{km} \cdot \mathrm{mol}^{-1}$ :

$$
\varepsilon=\frac{k \pi d^{2} l A \ln 10 \cdot 10^{-5}}{4 n_{\mathrm{ads}} x}=10.85 \cdot 10^{-7} \frac{A}{n_{\mathrm{ads}}} .
$$

Используя формулу (7), был рассчитан коэффициент экстинкции $\varepsilon(\mathrm{CO})$ с использованием интегральной интенсивности обеих полос $A_{\text {sum }}$ (табл. 1) для всех рассмотренных покрытий. При самых малых покрытиях $(\theta=0.002)$ получено $\varepsilon$, равное $110.6 \mathrm{~km} \cdot \mathrm{mol}^{-1}$, что соответствует коэффициенту экстинкции только для высокочастотной полосы при $2210 \mathrm{~cm}^{-1}$ (рис. 2,b). В то же время значение $\varepsilon$, вычисленное усреднением величин прироста интегральной интенсивности и соответствующего количества адсорбированных молекул при покрытиях больших, чем 0.02 (табл. 1), оказалось равным $60.3 \mathrm{~km} \cdot \mathrm{mol}^{-1}$, что соответствует коэффициенту экстинкции низкочастотной полосы при $2193 \mathrm{~cm}^{-1}$ (рис. 2, $b$ ).

В табл. 2 приведены коэффициенты экстинкции СО в газовой фазе и в адсорбированном состоянии на поверхности оксидов титана, цинка и кремния, взятые из литературы и рассчитанные для исследуемой в настоящей работе системы $\mathrm{CO} / \mathrm{TiO}_{2}$.

Как известно, интегральный коэффициент экстинкции $\varepsilon$ может быть выражен через производную дипольного момента $\mu$ по межъядерному расстоянию $r$ вдоль связи С-O [26]:

$$
\varepsilon=\left(\frac{N_{\mathrm{A}} \pi}{3 c^{2} m_{R}}\right)\left(\frac{d \mu}{d r}\right)^{2},
$$

где $N_{\mathrm{A}}$ - число Авогадро, $c$ - скорость света, $m_{R}-$ приведенная масса молекулы СО. При адсорбции изменения коэффициента экстинкции СО и, следовательно, интенсивности полосы поглощения СО по сравнению с таковыми для молекулы в газовой фазе определяется изменением величины $\frac{d \mu}{d r}$. Для свободной молекулы эта величина равна $3.1 \mathrm{D} / \AA$ [34]. Значение производной дипольного момента по расстоянию для СО в адсорбированном состоянии для исследуемой системы в литературе отсутствует, что делает затруднительным интерпретацию полученных экспериментальных данных. Вместе с тем рассчитанный коэффициент экстинкции $\mathrm{CO}$, адсорбированного на более сильных центрах, значительно выше значения для газовой фазы, в то время как таковой для $\mathrm{CO}$ на более слабых центрах сравним с ним по величине (табл. 2).

В работе [35] была теоретически исследована адсорбция CO на кластере MgO методом LCGTO-LDF. Кластерная модель предсказала примерно двукратное увеличение интенсивности ИК полосы адсорбированного СО при адсорбции углеродным концом на катионах магния. Несмотря на преобладание электростатического взаимодействия благодаря $\pi$-донированию, расчеты показали существенное влияние вклада $\pi$-донирования в результирующую величину $\frac{d \mu}{d r}$. Интересно отметить, что рассчитанные $\varepsilon$ для системы $\mathrm{CO} / \mathrm{MgO}$ значительно превышают рассчитанную $\varepsilon(\mathrm{CO})$ для газовой фазы. Очевидно, что для детального исследования влияния адсорбции на коэффициент экстинкции СО для системы $\mathrm{CO} / \mathrm{TiO}_{2}$ необходимо проведение аналогичных расчетов. 
Таблица 2. Значения коэффициента экстинкции моноксида углерода в газовой фазе и в адсорбированном состоянии на поверхности некоторых оксидов с указанием положения максимумов соответствующих полос поглощения

\begin{tabular}{|c|c|c|c|c|c|}
\hline № & Система & $\begin{array}{c}\text { Условия } \\
\text { эксперимента }\end{array}$ & $\begin{array}{l}v(\mathrm{CO}) \\
\mathrm{cm}^{-1}\end{array}$ & $\begin{array}{c}\varepsilon(\mathrm{CO}), \\
\mathrm{km} \cdot \mathrm{mol}^{-1}\end{array}$ & Ссылка \\
\hline 1 & $\begin{array}{c}\text { СО } \\
\text { в газовой фазе }\end{array}$ & $20^{\circ} \mathrm{C}$ & $\begin{array}{l}2143 \\
2143\end{array}$ & $\begin{array}{l}54 \\
65.5\end{array}$ & $[26]]^{*}$ \\
\hline 2 & $\mathrm{CO} / \mathrm{ZnO}$ (Kadox) & $25^{\circ} \mathrm{C}$ & $\begin{array}{l}2212 \\
2200 \\
2187\end{array}$ & $\begin{array}{l}90 \\
56 \\
18\end{array}$ & [26] \\
\hline 3 & $\mathrm{CO} / \mathrm{SiO}_{2}$ (aerosol) & \multirow{2}{*}{$\begin{array}{c}-196^{\circ} \mathrm{C} \\
n_{\mathrm{ads}} \approx 100-250 \\
\mu \mathrm{mol} / \mathrm{g}\end{array}$} & 2157 & 16 & \multirow[b]{2}{*}[6]{} \\
\hline 4 & $\mathrm{CO} / \mathrm{TiO}_{2}$ (Degussa P25) & & $\begin{array}{l}2210 \\
2190 \\
2178\end{array}$ & $\begin{array}{r}2 \\
8 \\
20\end{array}$ & \\
\hline 5 & $\mathrm{CO} / \mathrm{TiO}_{2}$ (анатаз) & \multirow{3}{*}{$\begin{array}{c}25^{\circ} \mathrm{C} \\
p(\mathrm{CO})=0-100 \\
\text { Torr }\end{array}$} & $\begin{array}{l}2206 \\
2187\end{array}$ & $\begin{array}{l}38 \\
26\end{array}$ & \multirow{3}{*}{ [5] } \\
\hline 6 & $\mathrm{CO} / \mathrm{TiO}_{2}$ (анатаз) & & 2195 & 31 & \\
\hline 7 & $\mathrm{CO} / \mathrm{TiO}_{2}$ (рутил) & & 2184 & 27 & \\
\hline 8 & $\begin{array}{c}\mathrm{CO} / \mathrm{TiO}_{2} \text { (Hombikat } \\
\text { UV100) }\end{array}$ & $\begin{array}{c}25^{\circ} \mathrm{C} \\
p(\mathrm{CO})=0-6 \text { Torr }\end{array}$ & $\begin{array}{l}2210 \\
2193\end{array}$ & $\begin{array}{r}110.6 \\
60.3\end{array}$ & $\begin{array}{c}\text { Настоящая } \\
\text { работа }\end{array}$ \\
\hline
\end{tabular}

Примечание. ${ }^{*}$ В работе [33] также представлены значения $\varepsilon$ для СО в газовой фазе, взятые из ранее опубликованных работ за период с 1951 по 1983 гг.

Анализ литературы показал, что имеется большой разброс значений $\varepsilon(\mathrm{CO})$ в адсорбированном состоянии для системы $\mathrm{CO} / \mathrm{TiO}_{2}$ (табл. 2). Очевидно, что при экспериментальном определении величина коэффициента экстинкции сильно зависит от условий проведения эксперимента, таких как температура системы, степень и равномерность покрытия адсорбатом, а также от параметров образца (фазовый состав, удельная поверхность, степень дегидратации поверхности). Так, при низкотемпературной адсорбции $\mathrm{CO}$ на $\mathrm{TiO}_{2}$ Degussa P25 значения коэффициентов экстинкции СО оказались равными 2,8 и $20 \mathrm{~km} \cdot \mathrm{mol}^{-1}$ для полос при 2210,2190 и $2178 \mathrm{~cm}^{-1}$ соответственно [6]. Эти величины меньше полученных $\varepsilon(\mathrm{CO})$ при адсорбции при комнатной температуре: 38 и $26 \mathrm{~km} \cdot \mathrm{mol}^{-1}$ [5]. Однако значения, полученные при комнатной температуре в настоящей работе, значительно превышают последние. Несмотря на схожие характеристики исследуемых образцов анатаза, следует отметить большую разницу в исследуемых диапазонах покрытий поверхности. Так, в работе [5] было исследовано всего два-три покрытия в диапазоне равновесного давления $\mathrm{CO}$ до 6 Torr, в то время как в настоящей работе указанный диапазон давлений исследовался детально (рис. 2 и 3 ).

На рис. 4 представлена зависимость $\varepsilon$ от покрытия поверхности молекулами $\mathrm{CO}$ для обеих полос. Хорошо видно, что $\varepsilon$ значительно уменьшается с увеличением степени заполнения поверхности в диапазоне малых $\theta$, что нельзя проследить в работе [5] в связи с проведением исследований при заметно бо́льших покрытиях.

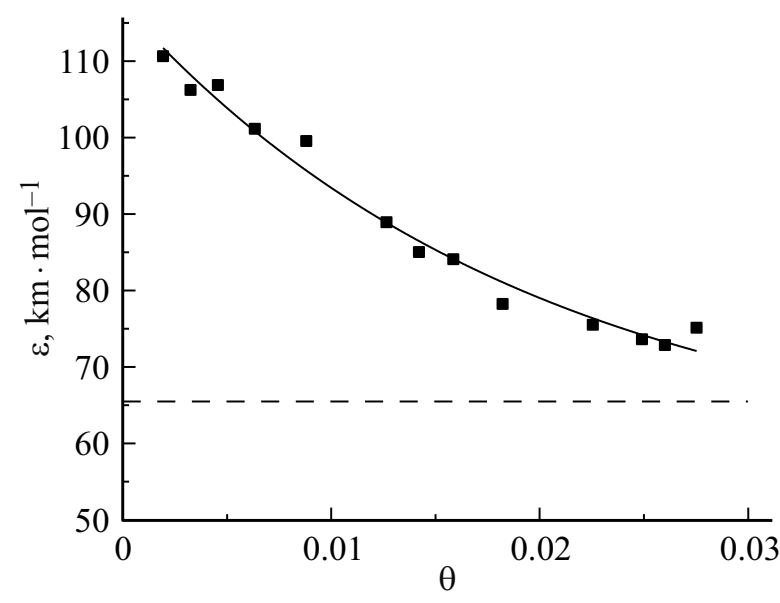

Рис. 4. Зависимость интегрального коэффициента экстинкции адсорбированного СО от покрытия. Аппроксимация экспериментальной зависимости с помощью логарифмической функции обозначена сплошной линией. Штриховой линией указано значение интегрального коэффициента экстинкции СО в газовой фазе при $20^{\circ} \mathrm{C}$, равное $65.5 \mathrm{~km} \cdot \mathrm{mol}^{-1}$ [33].

Анализ динамики изменения полученных спектров при увеличении покрытия, представленных на рис. 2, позволяет утверждать, что сначала происходит заполнение более сильных центров адсорбции, соответствующих высокочастотной полосе адсорбированного $\mathrm{CO}$, а затем заполняются центры, ответственные за появление низкочастотной полосы. Таким образом, значения коэф- 
фициента экстинкции, полученные из анализа спектров при малых покрытиях, должны быть практически одинаковыми до тех пор, пока происходит заполнение более сильных центров. После достижения насыщения интенсивности полосы при $2210 \mathrm{~cm}^{-1}$ начинается рост второй, низкочастотной компоненты при $2193 \mathrm{~cm}^{-1}$, что приводит к постепенному уменьшению результирующего коэффициента экстинкции до значений $73-75 \mathrm{~km} \cdot \mathrm{mol}^{-1}$ (рис. 2,b). Следовательно, можно сделать вывод, что на зависимости интегрального коэффициента экстинкции $\varepsilon$ адсорбированного СО от покрытия на рис. 4 в области самых низких покрытий должно наблюдаться „плато“, в пределах которого величина $\varepsilon$ практически не меняется. Однако в реальности такой области не наблюдается.

В связи с тем, что, как нами показано выше, энергетическая неоднородность поверхности исследуемого образца носит экспоненциальный характер, а теплота адсорбции в этом случае изменяется по логарифмическому закону с увеличением степени заполнения [19], экспериментальная зависимость $\varepsilon=f(\theta)$ была аппроксимирована с помощью логарифмической функции. Как видно из рис. 4, аппроксимирующая кривая хорошо описывает экспериментальные данные $\left(R_{\text {adj }}^{2}=0.98\right)$, что подтверждает наблюдаемый факт значительного уменьшения $\varepsilon$ с покрытием в области малых $\theta$. При дальнейшем увеличении покрытия эта зависимость имеет невыраженный характер, что и наблюдалось авторами в работе [5].

Хорошо известно, что переход из газовой в конденсированную фазу (например, жидкость, адсорбированное состояние или криоматрицы) всегда сопровождается изменением интенсивности полос в спектрах поглощения. Данный факт можно продемонстрировать на примере коэффициентов экстинкции СО в газовой фазе $\left(65.5 \mathrm{~km} \cdot \mathrm{mol}^{-1}\right)$ [33] и в аргоновой криоматрице $\left(94 \mathrm{~km} \cdot \mathrm{mol}^{-1}\right)[26]$.

Известно, что по мере увеличения показателя преломления среды эффект изменения интенсивности полос поглощения возрастает [36]. В этих случаях для гомогенных конденсированных систем широко применяется подход, согласно которому напряженность поля зондирующей световой волны изменяется при взаимодействии с конденсированной средой, что не наблюдается для газовой фазы. Для учета влияния этого изменения на интенсивность регистрируемых полос вводится так называемая поправка на поле Лоренца:

$$
L(n)=\frac{\left(n^{2}+2\right)^{2}}{9 n},
$$

где $n$ - показатель преломления среды.

Найденное значение $L(n)$ позволяет скорректировать интенсивность полосы для молекул в газовой фазе с учетом влияния конденсированной среды:

$$
\varepsilon_{\text {corr }}(\mathrm{CO})=L(n) \varepsilon\left(\mathrm{CO}_{\mathrm{g}}\right),
$$

где $\varepsilon_{\text {corr }}(\mathrm{CO})$ - скорректированный коэффициент экстинкции СО в конденсированной среде с учетом поправ- ки на поле Лоренца, $\varepsilon\left(\mathrm{CO}_{\mathrm{g}}\right)-$ коэффициент экстинкции СО в газовой фазе.

При изучении взаимодействия молекул с поверхностью твердых тел к гетерогенным системам „адсорбат-адсорбент“ также может быть применен описанный выше подход, применяемый обычно для гомогенных сред. В нашем случае адсорбент представляет собой мелкодисперсный порошок диоксида титана, спрессованный в тонкую таблетку, а адсорбат - молекулы моноксида углерода, равномерно распределенные по поверхности частиц, составляющих эту таблетку. Анализ микроскопического изображения прессованного образца (рис. 1) позволил оценить средние размеры пустот между частицами адсорбента, которые оказались равны 5-10 nm. Таким образом, можно считать, что при комнатной температуре параметры исследуемого образца позволяют молекулам адсорбата достаточно свободно диффундировать между частицами порошкообразного образца и равномерно адсорбироваться на их поверхности.

Эффективный показатель преломления исследуемого порошкообразного материала отличается от такового у монокристалла. Используя формулу Лорентц-Лоренца, связывающую плотность образца и его показатель преломления:

$$
\frac{n^{2}-1}{n^{2}+1}=\frac{4}{3} \pi \alpha \rho
$$

где $\alpha$ и $\rho-$ поляризуемость и плотность среды, нами была определена величина эффективного показателя преломления исследуемого поликристаллического образца с помощью соотношения

$$
\frac{\left(\frac{n_{1}^{2}-1}{n_{1}^{2}+1}\right)}{\left(\frac{n_{2}^{2}-1}{n_{2}^{2}+1}\right)}=\frac{\rho_{1}}{\rho_{2}},
$$

где $\rho_{1}$ и $\rho_{2}-$ плотности монокристаллического и дисперсного диоксида титана фазы анатаз, $\rho_{1}=3.9 \mathrm{~g} \cdot \mathrm{cm}^{-3}$, $n_{1}=2.561$ [37]. Плотность исследуемого прессованного образца определили из известных линейных размеров образца и его массы: $\rho_{2}=1.47 \mathrm{~g} \cdot \mathrm{cm}^{-3}$.

Подставляя в формулу (9) рассчитанное значение $n_{2}$, равное 1.4 , получаем величину поправки $L(n)=1.2446$; по формуле (10) определяем скорректированный коэффициент экстинкции $\mathrm{CO} \varepsilon_{\mathrm{corr}}(\mathrm{CO})=81.5 \mathrm{~km} \cdot \mathrm{mol}^{-1}$, используя $\varepsilon\left(\mathrm{CO}_{\mathrm{g}}\right)=65.5 \mathrm{~km} \cdot \mathrm{mol}^{-1}$ [33]. Найденное значение оказывается несколько меньше максимального значения в адсорбированном состоянии, полученного из закона Бугера-Ламберта-Бера $\left(110.6 \mathrm{~km} \cdot \mathrm{mol}^{-1}\right.$, табл. 2). Можно сделать вывод, что слабая адсорбция СО, наблюдаемая в экспериментах, не приводит к существенному изменению $\varepsilon$.

Однако приведенное выше экспериментально полученное максимальное значение $\varepsilon$ было вычислено в предположении, что длина оптического пути равна толщине образца. В реальности длина оптического пути из-за многократных отражений между частицами внутри 
Таблица 3. Рассчитанные коэффициенты экстинкции СО, адсорбированного на поверхности диоксида титана, для разных значений оптического пути при различных покрытиях

\begin{tabular}{c|c|c|c|c|c|c}
\hline \multirow{2}{*}{ № } & \multirow{2}{*}{$\theta$} & \multicolumn{4}{|c}{$\varepsilon\left(\mathrm{km} \cdot \mathrm{mol}^{-1}\right)$ для оптического пути $x$, равного } \\
\cline { 3 - 7 } & & $1 l$ & $1.25 l$ & $1.5 l$ & $1.75 l$ & $2 l$ \\
\hline 1 & 0.002 & 110.6 & 88.5 & 73.7 & 63.2 & 55.3 \\
2 & 0.013 & 88.9 & 71.1 & 59.3 & 50.8 & 44.5 \\
3 & 0.026 & 72.9 & 58.3 & 48.6 & 41.7 & 36.5
\end{tabular}

прессованного образца имеет большую величину, которую экспериментально невозможно точно определить. Решение этой задачи требует построения специальных моделей, учитывающих (помимо размера и формы частиц) плотность упаковки, спектральный состав анализируемого света. В настоящей работе не ставилась цель решить данную задачу. Однако нами было показано поведение $\varepsilon$ с увеличением длины оптического пути. Для этого в качестве оптического пути в уравнении Бугера-Ламберта-Бера (5) брались значения в диапазоне от одной до двух толщин образца $(I)$. Соответствующие данные приведены в табл. 3.

Из таблицы видно, что при увеличении оптического пути на 25\% значение при наименьшем покрытии приближается к откорректированному значению $\varepsilon$ для конденсированной среды. Отсюда можно сделать вывод, что $\varepsilon$ для слабо адсорбированных молекул $\mathrm{CO}$ на оксиде титана несущественно отличается от значения для газовой фазы. Очевидно, что более корректным будет сравнение коэффициентов экстинкции $\mathrm{CO}$ в адсорбированном состоянии, рассчитанных с использованием закона Бугера-Ламберта-Бера, для гетерогенных систем со схожими рассеивающими параметрами, а не с коэффициентом экстинкции СО в газовой фазе. При этом при проведении экспериментов желательно использовать образцы с примерно одинаковой толщиной.

\section{Заключение}

Методами ИК спектроскопии и волюметрии изучена адсорбция моноксида углерода на поверхности $\mathrm{TiO}_{2}$ (анатаз) при комнатной температуре. Полученные экспериментальные данные описываются изотермой адсорбции Фрейндлиха, характеризующей адсорбцию на экспоненциально-неоднородной поверхности. Присутствие двух типов центров адсорбции на поверхности исследуемого образца также подтверждается наличием двух ИК полос при $2210 \mathrm{~cm}^{-1}$ и $2193 \mathrm{~cm}^{-1}$, принадлежащих валентным колебаниям адсорбированного СО. Рассчитанные значения теплоты адсорбции для двух типов центров $\mathrm{Ti}^{4+}\left(44.0\right.$ и $\left.35.5 \mathrm{~kJ} \cdot \mathrm{mol}^{-1}\right)$ соответствуют слабой адсорбции. Теплота адсорбции уменьшается по логарифмическому закону с покрытием в диапазоне $0.002-0.03$.

Коэффициент экстинкции молекул СО в адсорбированном состоянии для гетерогенной системы $\mathrm{CO} / \mathrm{TiO}_{2}$ рассчитан с использованием закона Бугера-Ламберта-Бера при условии равенства оптического пути толщине образца. При минимальном покрытии $\varepsilon=110.6 \mathrm{~km} \cdot \mathrm{mol}^{-1}$, что соответствует молекулам $\mathrm{CO}$, адсорбированным на более сильных центрах. При больших покрытиях удалось определить коэффициент экстинкции для молекул СО, адсорбированных на более слабых центрах, равный $60.3 \mathrm{~km} \cdot \mathrm{mol}^{-1}$. Обнаруженная зависимость исследуемого параметра от величины покрытия поверхности адсорбатом объясняется энергетической неоднородностью поверхности.

Анализ работ, посвященных определению коэффициентов экстинкции СО в адсорбированном состоянии, позволил определить факторы, влияющие на $\varepsilon$. Степень покрытия поверхности адсорбатом, зависящая от температуры системы и давления адсорбата, определяет коэффициент экстинкции для одного и того же образца, особенно при малых покрытиях, как продемонстрировано в настоящей работе.

Продемонстрирован вклад поля зондирующей световой волны прибора на коэффициент экстинкции адсорбата в конденсированной системе $\mathrm{CO} / \mathrm{TiO}_{2}$. Учет поправки на поле Лоренца среды-адсорбента позволил получить скорректированное значение коэффициента экстинкции $\mathrm{CO}$, равное $81.5 \mathrm{~km} \cdot \mathrm{mol}^{-1}$, что выше $\varepsilon(\mathrm{CO})$ в газовой фазе и согласуется с $\varepsilon$ для $\mathrm{CO}$ в адсорбированном состоянии, рассчитанными по уравнению Бугера-Ламберта-Бера.

Применимость закона Бугера-Ламберта-Бера для определения коэффициента экстинкции СО в гетерогенных системах ограничивается несоответствием толщины образца оптическому пути из-за рассеивающей способности адсорбента, зависящей от размера его частиц и плотности их упаковки в исследуемом образце. Таким образом, использование этого подхода при определении коэффициентов экстинкции адсорбированного СО и для сравнения полученных значений для разных материалов правомерно для образцов со схожими рассеивающими свойствами и близкими толщинами.

\section{Благодарности}

Настоящая работа выполнена при финансовой поддержке Санкт-Петербургского государственного университета в рамках проекта „Приглашение ведущего ученого для создания лаборатории“ (Pure ID 73032813). Экспериментальные данные были получены в ходе выполнения гранта РФФИ (№ 18-03-00855). Авторы также благодарят ресурсный центр „Нанотехнология“ Научного парка СПбГУ за помощь в проведении микроскопического исследования образца. 


\section{Конфликт интересов}

Авторы заявляют, что у них нет конфликта интересов.

\section{Список литературы}

[1] Lamberti C., Zecchina A., Groppo E., Bordiga S. // Chem. Soc. Rev. 2010. V. 39. P. 4951-5001. doi 10.1039/C0CS00117A

[2] Yang Ch., Wöll C. // Advances in Physics. V. 2. I. 2. P. 373-408. doi 10.1080/23746149.2017.1296372

[3] Tsyganenko A.A., Storozhev P.Yu., Otero Areán C. // Kinet. and Catal. 2004. V. 45(4). P. 530-540. doi 10.1023/B:KICA.0000038081.43384.56

[4] Hadjiivanov K.I., Vaysilov G.N. // Adv. Catal. 2002. P. 307-511. doi 10.1016/S0360- 0564(02)47008-3

[5] Kondratieva E.V., Manoilova O.V., Tsyganenko A.A. // Kinet. Catal. 2008. V. 49(3). P. 451-456. doi 10.1134/S002315840803021X

[6] Morterra C., Garrone E., Bolis V., Fubini B. // Spectrochim. Acta. 1987. V. 43A(12). P. 1577-1581. doi 10.1016/S0584-8539(87)80051-X

[7] Bulanin K.M., Bahnemann D.W., Rudakova A.V. // Rev. Sci. Instrum. 2019. V. 90(10). ID 105113. doi 10.1063/1.5100510

[8] Morterra C., Ghiotti G., Garrone E., Fisicaro E. // J. Chem. Soc. Faraday Trans. 1980. V. 76. P. 2102-2113. doi 10.1039/F19807602102

[9] Morterra C., Bolis V., Fisicaro E. // Colloid. Surf. 1989. V. 41. P. 177-188. doi 10.1016/0166-6622(89)80051-4

[10] Mino L., Ferrari A.M., Lacivita V., Spoto G., Bordiga S., Zecchina A. // J. Phys. Chem. C. 2011. V. 115. P. 7694-7700. doi 10.1021/jp2017049

[11] Hush N.S., Williams M.L. // J. Molec. Spectrosc. 1974. V. 50. P. 349-368. doi 10.1016/0022-2852(74)90241-0

[12] Hadjiivanov K.I., Vayssilov G.N. // Adv. Catal. 2002. P. 307-511.

[13] Pacchioni G., Ferrari A.M., Bagus P.S. // Surf. Sci. 1996. V. 350. P. $159-175$.

[14] Linsebigler A., Lu G., Yates J.T., Jr. // J. Chem. Phys. 1995. V. 103(21). P. 9438-9443. doi 10.1063/1.470005

[15] Артемьев Ю.М., Рябчук В.К. Введение в гетерогенный катализ. Уч. пособие. СПб.: Изд-во СПбГУ, 1999. 304 с.

[16] Langmuir I. // J. Am. Chem. Soc. 1918. V. 40(9). P. 1361-1403. doi 10.1021/ja02242a004

[17] Freundlich H. Colloid and Capillary Chemistry. London: Methuen Co., Ltd., 1926. 883 p.

[18] Dabrowski A. // Adv. Colloid Interface Sci. 2001. V. 93(1-3). P. 135-224. doi 10.1016/S0001-8686(00)00082-8

[19] Foo K.Y., Hameed B.H. // Chem. Eng. J. 2010. V. 156. P. 2-10. doi 10.1016/j.cej.2009.09.013

[20] Soltanov R.I. // Kinet. Catal. 1990. V. 31(2). P. 376-380.

[21] Паукштис E.A. // Оптическая спектроскопия в адсорбции и катализе. Применение к ИК-спектроскопии. Уч. пособие. Новосибирск: Изд-во Института катализа СО РАН, 2010. $55 \mathrm{c}$.

[22] Paukshtis E.A., Yurchenko E.N. // Russ. Chem. Rev. 1983. V. 52(3). P. 242-258.

[23] Dohnalek Z., Kim J., Bondarchuk O., White J.M., Kay B.D. // J. Phys. Chem. B. 2006. V. 110. P. 6229-6235. doi $10.1021 /$ jp0564905
[24] Lin L., Shi Z., Yan L., Tao H., Yao L., Li S., Xie K., Huang J., Zhang Z. // Polyhedron. 2020. V. 191. ID 114814. doi 10.1016/j.poly.2020.114814

[25] Setvin M., Buchholz M., Hou W., Zhang C., Stöger B., Hulva J., Simschitz T., Shi X., Pavelec J., Parkinson G.S., Xu M., Wang Y., Schmid M., Wöll C., Selloni A., Diebold U. // J. Phys. Chem. C. 2015. V. 119. P. 21044-21052. doi 10.1021/acs.jpcc.5b07999

[26] Seanor D.A., Amberg C.H. // J. Chem. Phys. 1965. V. 42(8). P. 2967-2970. doi 10.1063/1.1703271

[27] Chen J., Garcia E.D., Oliviero L., Maugé F. // J. Catal. 2015. V. 332. P. 77-82. doi 10.1016/j.jcat.2015.09.005

[28] Tarach K., Gora-Marek K., Chrzan M., Walas S. // J. Phys. Chem. C. 2014. V. 118. P. 23751-23760. doi $10.1021 / \mathrm{jp} 506820 \mathrm{v}$

[29] Zholobenko V., Freitas C., Jendrlin M., Bazin P., Travert A., Thibault-Starzyk F. // J. Catal. 2020. V. 385. P. 52-60. doi 10.1016/j.jcat.2020.03.003

[30] Badri A., Binet C., Lavalley J.-C. // J. Chem. Soc. Faraday Trans. 1997. V. 93(6). P. 1159-1168. doi 10.1039/a606628c

[31] Morterra C., Magnacca G., Bolis V. // Catal. Today. 2001. V. 70. P. 43-58. doi 10.1016/S0920-5861(01)00406-0

[32] Tan C.-D., Ni J. // J. Chem. Eng. Data. 1997. V. 42. P. 342-345. doi 10.1021/je960211c

[33] Kim K. // J. Quant. Spectrosc. Radiat. Transf. 1983. V. 30(5). P. 413-416. doi 10.1016/0022-4073(83)90104-8

[34] Billingsley F.P., Krauss M. // J. Chem. Phys. 1974. V. 60. P. 4130. doi $10.1063 / 1.1680880$

[35] Neyman K.M., Rösch N. // Surface Science. 1993. V. 297. P. 223-234. doi 10.1016/0039-6028(93)90266-M

[36] Волькенштейн М.В. Строение и физические свойства молекул. М.; Л.: Изд-во Академии наук СССР, 1955. 637 с.

[37] Электронный ресурс. Режим доступа: http://webmineral.com/data/Anatase.shtml\#.YJGgcLUzaUk 